\title{
Numerical Investigation on the Leakage Characteristics of Brush Seals Based on Fluid-Structure Interaction
}

\author{
Yitong Wang \\ School of Civil Engineering, University of Science and Technology LiaoNing, Anshan 114051 China \\ 995031734@qq.com
}

Keywords: Brush seals; Fluid-structure interaction; Flow field characteristic; Leakage characteristic

\begin{abstract}
This paper investigates the leakage characteristics of brush seals including the flow field characteristic and the effects of structural parameters on the leakage characteristic, which was discussed based on three-dimensional (3-D) computational model of brush seal, two-way fluid-structure interaction. As illustrated in the analysis of the leakage characteristics of brush seals, (1) the leakage with the influence of bristle deflection is closer to the results of experiment relative to that without bristle deflection, which validate the developed 3-D computational model to be more reasonable; (2) the flow field characteristics (pressure and velocity) of brush seal are revealed reasonably; (3) with the increasing of the height of backing plate fence, the clearance of brush wire and the axial clearance between brush bristle and back plate, the leakage factor rises and then reaches to a stable value when the clearance of brush is larger than $0.3 \mathrm{~mm}$; moreover, (4) with the increase of brush wire diameter, the leakage factor decreases firstly and then tends to stabilization. The efforts of this paper provide a useful numerical method to clearly understand the leakage characteristics of brush seal, which is beneficial to improve the design of brush seals.
\end{abstract}

\section{Introduction}

As a new and promising dynamic sealing technology brush seals has widely used in turbomachinery over the last decade [1-2]. According to the investigations, brush seals significantly improve the performance and efficiency of turbines over the conventional labyrinth seals[3]. The brush seals consist of front plate, backing plate and spaced bristle pack clamped between the two parts, as illustrated in Fig. 1. The flexible bristle pack has a typical lay angle between 30 degrees and 60 degrees in the direction of rotor.

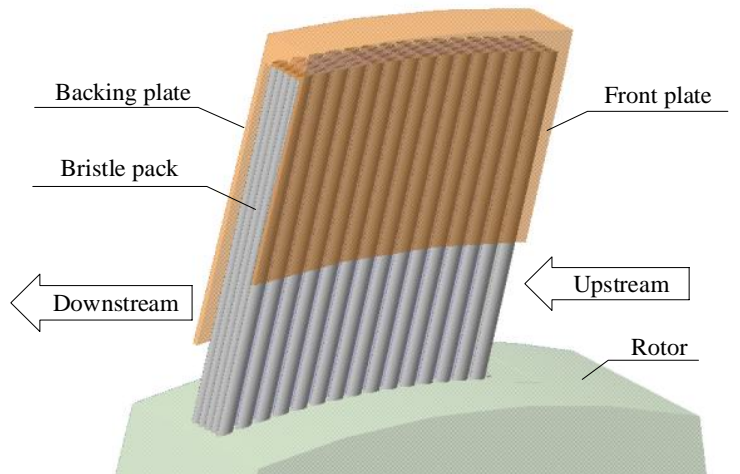

Figure 1. Schematic diagram of brush seal structure

In recent years, many efforts have been focused on numerically investigate the leakage flow characteristics of brush seals. For example, Chew [4] and Hogg [5] simulated the leakage flow in the brush seals by the non-darcian porous medium approach. Dogu [6,7] and Aksit [8,9] employed the modified bulk porous medium approach to evaluate the effects of the front and backing plate configurations on the flow fields of brush seals. Qiu and Li [10] discussed the leakage flow of two kinds of brush seals with the porous medium model. Most of the studied literatures analyzed the leakage flow characteristics of brush seals based on the simplified porous medium model, and did not fluid-solid interaction(FSI). 
Practical operation and studies have shown that the deflection of flexible bristle pack generates due to flowing pressure and thereby influences the flow characteristics. Under this circumstance, FSI analysis is implemented by two-way coupling iterative algorithm. The objective of this paper is to present the investigations of brush seal comprising of the flow field characteristics and the effect of structural parameters on the leakage characteristic. The efforts of this study provide the theoretical basis for the design of brush seal structure.

\section{Numerical Methodologies}

In this section, the basic theory and methodology related to the investigation on the leakage characteristics of brush seals based on fluid-structure interaction were discussed containing the governing equations of fluid and solid analyses, the principle of two-way FSI, computational domain and boundary conditions, and grid technology as well.

Governing Equations. Fluid governing equations. Suppose that the leakage flow is turbulent and incompressible. It may be deduced by the pressure difference across brush seals. The governing equations of an incompressible flow include continuity equation and Naiver-Stokes(N-S) (momentum conservation) equations. Continuity equation and $\mathrm{N}-\mathrm{S}$ equation are explained by Eq.(1) and Eq.(2) [11], i.e.,

$$
\begin{aligned}
& \frac{\partial \rho}{\partial t}+\nabla \cdot(\rho \boldsymbol{U})=0 \\
& \left\{\begin{array}{l}
\frac{\partial(\rho \boldsymbol{u})}{\partial t}+\nabla \cdot(\rho \boldsymbol{U} \boldsymbol{u})=\nabla \cdot(\Gamma \operatorname{grad} \boldsymbol{u})-\frac{\partial \boldsymbol{p}}{\partial x}+S_{u} \\
\frac{\partial(\rho \boldsymbol{v})}{\partial t}+\nabla \cdot(\rho \boldsymbol{U v})=\nabla \cdot(\Gamma \operatorname{grad} \boldsymbol{v})-\frac{\partial \boldsymbol{p}}{\partial y}+S_{v} \\
\frac{\partial(\rho \boldsymbol{w})}{\partial t}+\nabla \cdot(\rho \boldsymbol{U} \boldsymbol{w})=\nabla \cdot(\Gamma \operatorname{grad} \boldsymbol{w})-\frac{\partial \boldsymbol{p}}{\partial z}+S_{w}
\end{array}\right.
\end{aligned}
$$

where $\nabla$-the divergence; t-time; $\rho$-density; $p$-pressure; $U$-velocity vector; $x, y$ and $z$-the directions in Cartesian space respectively; $u, v$ and $w$-the components of the velocity vector $U$ along $x, y$ and $z$ directions in Cartesian space, respectively; $\Gamma$-eddy diffusivity; $S_{u}, S_{v}$ and $S_{w^{-}}$-source terms of momentum equation along $x, y$ and $z$ directions in Cartesian space, respectively.

To improve the accuracy for turbulence flows in brush seals, the RNG k- $\varepsilon$ model is used, [12] i.e.

$$
\begin{aligned}
& \frac{\partial(\rho k)}{\partial t}+\frac{\partial\left(\rho \boldsymbol{U}_{i} k\right)}{\partial x_{i}}=\frac{\partial}{\partial x_{j}}\left[\alpha_{k} \mu_{e f f} \frac{\partial k}{\partial x_{j}}\right]+G_{k}+\rho \varepsilon \\
& \frac{\partial(\rho \varepsilon)}{\partial t}+\frac{\partial\left(\rho \boldsymbol{U}_{i} \varepsilon\right)}{\partial x_{i}}=\frac{\partial}{\partial x_{j}}\left[\alpha_{\varepsilon} \mu_{e f f} \frac{\partial \varepsilon}{\partial x_{j}}\right]+C_{1 \varepsilon}^{*} \frac{\varepsilon}{k} G_{k}-1.68 \rho \frac{\varepsilon^{2}}{k}
\end{aligned}
$$

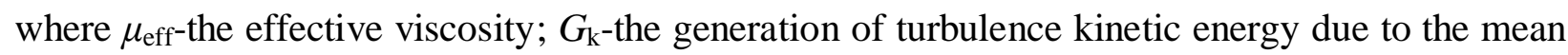
velocity gradients; $k$-turbulent kinetic energy; $\varepsilon$-turbulent dissipation rate; $\alpha_{\mathrm{k}}, \alpha_{\varepsilon}$-the inverse effective Prandtl numbers for $k$ and $\varepsilon$, respectively; $C_{1 \varepsilon}^{*}$-the model constants; $U_{\mathrm{i}}$ and $U_{\mathrm{j}}$-the tensor forms of $U ; x_{\mathrm{i}}$ and $x_{\mathrm{j}}$ - the tensor forms of $x$. ${ }_{\mathrm{i}}$ and $\mathrm{j}_{\mathrm{j}}$ the tensor indicators.

Solid governing equations. Bristles structural domain is divided into free finite element mesh by using dynamic analysis module in Workbench. The equation of structural dynamics is shown as follows

$$
[\boldsymbol{M}] \ddot{\boldsymbol{x}}+[\boldsymbol{C}] \dot{\boldsymbol{x}}+[\boldsymbol{K}] \boldsymbol{x}=P_{i}(t)
$$

in which $[M]$-mass matrix; $[C]$-damping matrix; $[K]$-stiffness matrix; $\ddot{x}, \dot{x}, x$-acceleration vector, velocity vector and displacement vector, respectively; $\mathrm{Pi}(\mathrm{t})$-the vector of loads changing with time. 
Two-way FSI Mechanism. For two-way FSI, the N-S equation, continuity equation and structural dynamic equation are solved synchronously, and MFS (multi-field solver) is used to realize the real-time exchange of information between flow field and structural field. The flow chat of two-way FSI is shown in Fig. 2. For the two-way FSI analysis of brush seals, the results from steady flow calculation were invoked. At the time Tn, the aerodynamic force acting on the bristles was calculated by using gas-dynamic models. Then the aerodynamic force on the bristles was acquired through the CFD calculation. The aerodynamic forces were transferred to the mechanical model of the brush bristle structure and caused the deflection of brush bristles. Meanwhile, the deflection of brush bristles influences the flow field distribution. The above analysis process was continuously executed until satisfying the aim of convergence. Lastly, the bristles locate on an equilibrium position determined by gas lifting force and elastic force of the bristles.

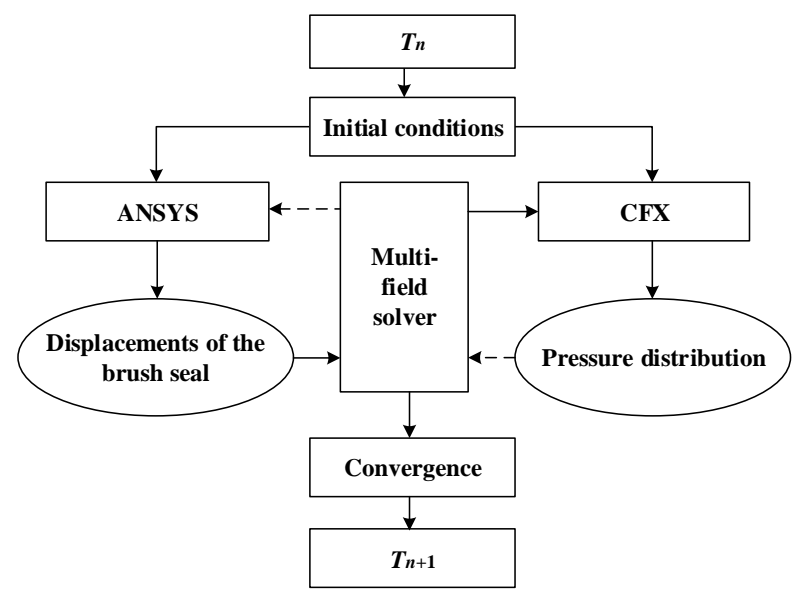

Figure 2. FSI simulations with two-way coupling

Computational Domain and Boundary Conditions. Computational domain. In this paper, the simplified model of the brush seal is shown in Fig. 3. Incompressible flow along axial direction was adopted in this simplified model.

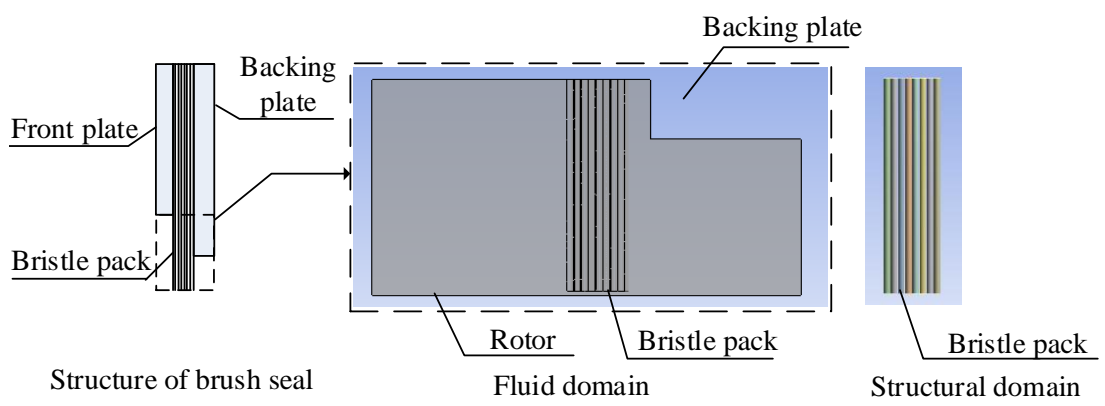

Figure 3. Computational domain of brush seal

Boundary conditions and mesh governing strategies. The FSI analysis was completed by adopting total pressure import, no slip solid wall boundary and wall function method. Two FSI interfaces are shown in Fig. 4. Therein, one was the fluid surface contacting with bristles. The other was the cylinder surface of bristles contacting with the fluid. Mesh deformation way of the FSI interfaces was motion. Residual convergence target of flow field calculation was 10-4. During transferring coupling data, relaxation factor was 0.75 and convergence criterion was $10-3$. 


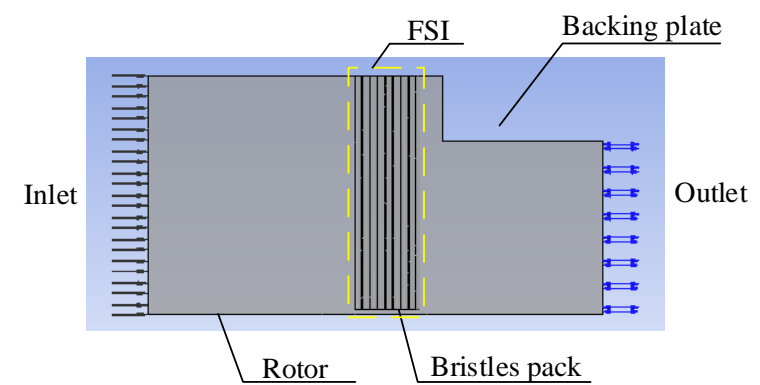

Figure 4. Sketch of FSI model boundary conditions

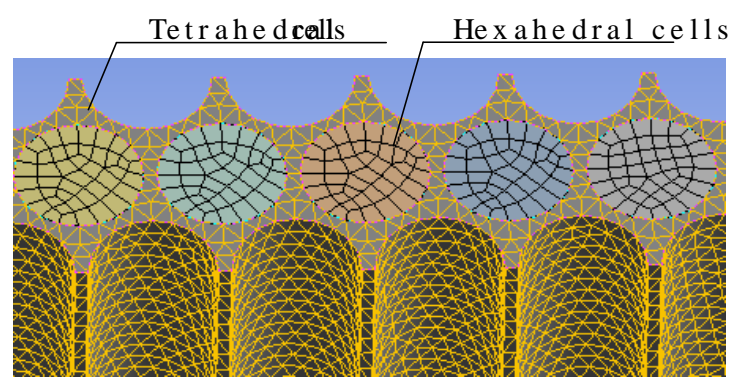

Figure 5. Meshing sketch of brush seals

The brush seals geometry contains the channels with high aspect ratio between bristles. Therefore, it is difficult that the flow feature in a small gap is captured by the fine mesh between bristles. Based on mesh generator in ICEM software, solid and flow domains were meshed into hexahedral and tetrahedral cells, respectively. A reasonable meshing with 490000 elements and 120000 elements for flow and structural domains respectively, was determined by the comparison of the results of FSI analyses under different mesh density of seal geometry. The gained meshing is shown in Fig. 5.

\section{Leakage Characteristic Analysis of Brush Seals}

Leakage Evaluation of Brush Seals with Bristle Deflection. In line with the above-mentioned theory, the leakage of brush seal, as a main performance parameter, was evaluated based on FSI analysis by the consideration with/without respect to bristle deflection. Moreover, thereby the results were compared with the experimental data from the document [13], which are shown in Fig. 6.

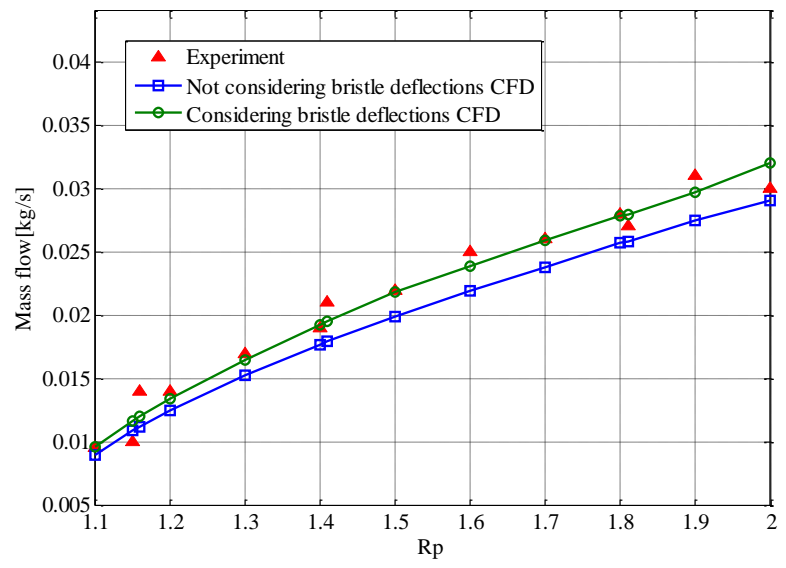

Figure 6. Comparison of experimental leakage with CFD results

As illustrated in Fig.6, the leakage for CFD analysis with the consideration of bristle deflection has the small error (about 5\% 8\%) and is closer to the experiment results than that without bristle deflection. It explains that bristle deflection greatly influence the leakage of brush seals at smaller inlet/outlet pressure ratio and the difference of the leakages with/without bristle deflection increases with the increasing inlet/outlet pressure ratio. The presented 3-D computational model of brush seals in two-way FSI analysis is demonstrated to be high precision for the prediction of seal flow characteristics.

Flow Distribution Characteristic Analysis of Brush Seals. From the FSI analysis, the pressure contours and velocity vectors on the brush seal are shown in Fig. 7. 

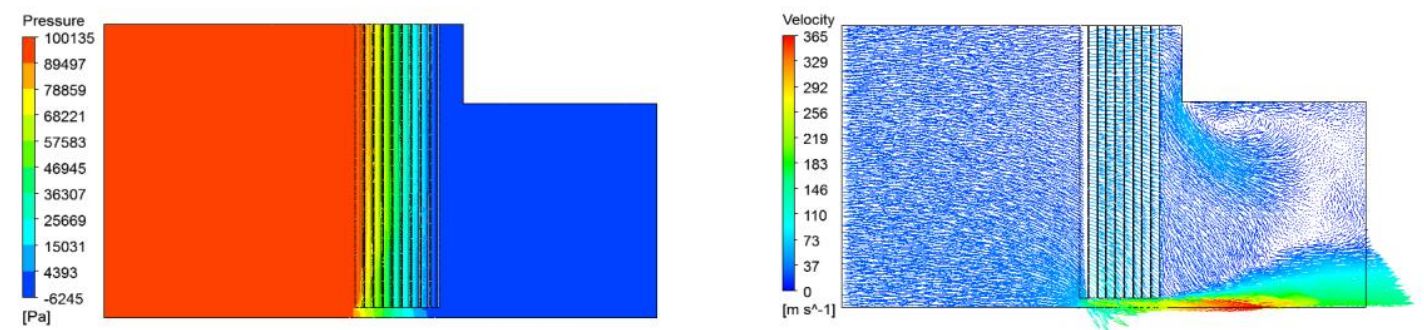

(a) Pressure distribution on radial cross section (b) Velocity vector distribution on radial cross section

Figure 7. Pressure and velocity vector distribution of the brush seal

From five kinds of color change across the bristles pack in Fig.7(a), it is illustrated that the pressure gradually drops from left to right on the bristles pack. As demonstrated in Fig.7(b), (1) the flow is smooth near the left of the seal. Due to the radial clearance between the bristles pack and the rotor, the flow approaches to this clearance with increasing velocity. (2) Because the axial flow is blocked by the bristles pack and backing plate, strong inward radial flow appears and then flow reaches to the downstream cavity (outlet). The fluid forms strong eddy at the corner of backing plate. (3) The maximum velocity $(365 \mathrm{~m} / \mathrm{s}$ ) occurs at the clearance between rotor and bristles pack.

Influence Analysis of Structural Parameters on Leakage Characteristics. The section separately evaluates the effect of the five structural parameters on leakage factor[14]. When one structural parameter is analyzed, the structural parameter is changed within its range (listed in Table 1) while other structural parameters are constant and their baseline values are selected in which the baseline value is explained by the real sizes of brush seal structure.

Table 1 Analyzed structural parameters with minimum, maximum, and baseline values

\begin{tabular}{|c|l|l|}
\hline No. & \multicolumn{1}{|c|}{ Parameters } & Parameter values (min, baseline, max) \\
\hline 1 & Backing plate fence height & $0.6-1.1-1.6-2.1-2.6-3.1-\underline{3.6}[\mathrm{~mm}]$ \\
2 & Axial distance & $0-0.1-0.2-0.3-0.4-\underline{0.5}-0.6-0.7-0.8[\mathrm{~mm}]$ \\
3 & The clearance of brush wire & $0.005-0.007-0.009-0.011-0.013-\underline{0.015}[\mathrm{~mm}]$ \\
4 & The diameter of brush wire & $0.07-0.09-0.11-0.13-\underline{0.15}-0.17-0.19[\mathrm{~mm}]$ \\
\hline
\end{tabular}

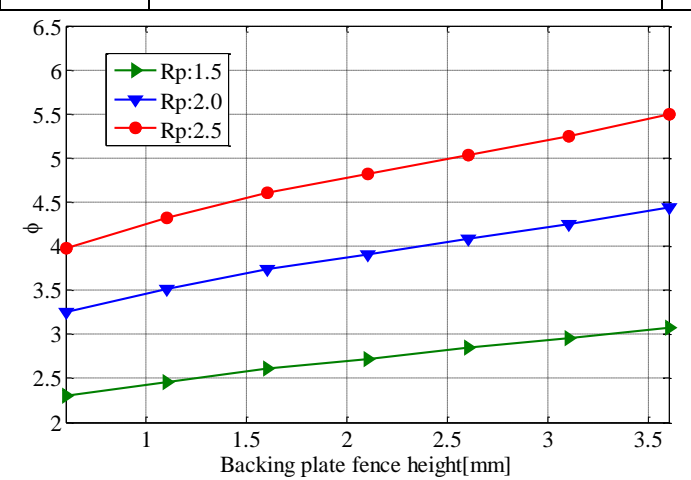

(a) Effect of the height of backing plate fence

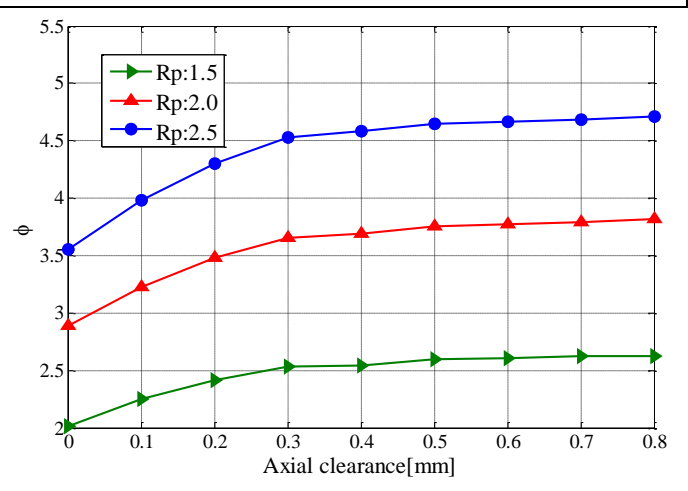

(b) Effect of the axial clearance of backing plate 


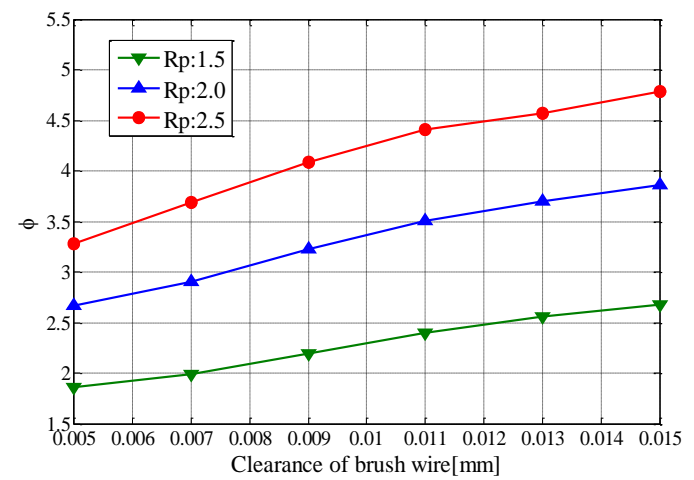

(c) Effect of the clearance of brush wire

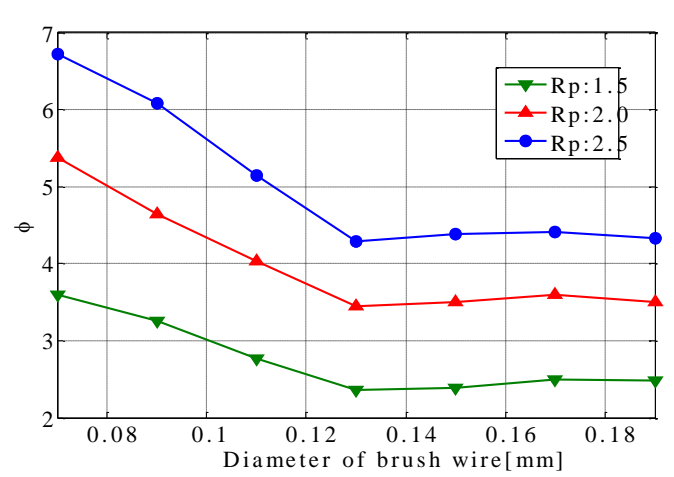

(d) Effect of the diameter of brush wire

Figure 8. The effect of five structural parameters on leakage factor

As revealed from Fig.8, for all structural parameters, the leakage factor almost raises with the increase of $\mathrm{Rp}$. With the rise of the height of the backing plate fence, the axial clearance of backing plate in range $[0 \mathrm{~mm}, 0.3 \mathrm{~mm}]$ and the clearance of brush wire, the leakage factor enlarges, meanwhile, reaches to a stable value at the axial clearance $0.3 \mathrm{~mm}$ under different Rp. For the diameters of brush wire, the leakage factor equably reduces in $[0.07 \mathrm{~mm}, 0.13 \mathrm{~mm}]$ and then is almost changeless during [0.13 mm, $0.19 \mathrm{~mm}$ ] for different $\mathrm{Rp}$.

\section{Conclusions}

The leakage characteristics of brush seals with bristles deflection are discussed based on moving grids technique and FSI approach from theoretical and numerical perspective, which includes the flow distribution characteristics of brush seals with the 3D computational model and the effect of structural parameters on leakage factor. Some conclusions are summarized as follows:

The leakage difference is changeless at smaller inlet/outlet pressure ratios whether bristle deflection is considered or not. However, the increase of inlet/outlet pressure ratio enlarges the leakage difference. The leakage for CFD analysis with the consideration of bristle deflection is closer to the experiment results than that without bristle deflection. Therefore, the presented 3-D computational model of brush seals with consideration of bristle deflection based on two-way fluid-structure interaction is more accurate for predicting the seal flow characteristics.

The pressure gradually drops from left to right on the bristle pack or across bristles; the flow approaches to radial clearance between the bristles pack and the rotor with increasing velocity; strong eddy occurs at the corner of backing plate; the maximum velocity of $365 \mathrm{~m} / \mathrm{s}$ appears in the clearance between rotor and bristle pack.

The leakage factor increases with the raise of both the height of backing plate fence and the clearance of brush wire. With the increasing axial clearance between the brush bristle and back plate, meanwhile, the leakage factor rises and then reaches to a steady value; with the increase of brush wire diameter, the leakage factor reduces firstly and then tends to stabilized.

\section{References}

[1] V.V. Kudriavtsev, M.J. Braun, Model development of the brush seal numerical simulation, J. Propuls Power. 12 (1) (1996) 193-201.

[2] R.E. Chupp, P. Nelson, Evaluation of brush seals for limited-life engines, J. Propuls Power. 9 (1) (1993) 113-118.

[3] F.E. Aslan-zada, V.A. Mammadov, F. Dohnal, Brush seals and labyrinth seals in gas turbine applications, J. Power. Energy. 227 (2) (2013) 216-230. 
[4] J.W. Chew, B.L. Lapworth, Mathematical modeling of brush seals, J. Heat Fluid Flow. 16 (6) (1995) 493-500.

[5] J.W. Chew, S.I. Hogg, Porosity modeling of brush seals, J. Tribol. 119 (4) (1997) 769-775.

[6] Y. Dogu, Investigation of brush seal flow characteristics using bulk porous medium approach, J. Eng. Gas Turbines Power. 127 (1) (2005) 136-144.

[7] Y. Dogu, M.F. Aksit, Effects of geometry on brush seal pressure and flow fields-part I front plate configurations, J. Turbomach. 128 (4) (2006) 367-378.

[8] Y. Dogu, M.F. Aksit, Effects of geometry on brush seal pressure and flow fields-part II backing plate configurations, J. Turbomach. 128 (4) (2006) 379-389.

[9] Y. Dogu, M.F. Aksit, Evaluation of flow behavior for clearance brush seals, J. Eng. Gas Turbines Power. 130 (1) (2008) 012507.

[10]B. Qiu, J. Li, Numerical investigations on the heat transfer behavior of brush seals using combined computational fluid dynamics and finite element method, J. Heat Transfer. 135 (12) (2013) 122601.

[11]L. F. Juan, B. Pascal, Three-dimensional fluid-structure interaction simulation with a hybrid RANS-LES turbulence model for applications in transonic flow domain, Aerosp. Sci. Technol. (49) (2016) 1-16.

[12] S.A. Orszag, V. Yakhot, Renormalization group analysis of turbulence. I .basic theory, J. Sci. Comput. 1 (1) (1986) 3-51.

[13] M. T. Turner, Experimental investigation and mathematical modelling of clearance brush seal, J. Eng. Gas Turbines Power. 120(3) (1998) 573-579.

[14]J. Ahmed, M. Gamal, Labyrinth seal leakage tests: tooth profile, tooth thickness and eccentricity effects, J. Eng. Gas Turbines Power. (130) (2008) 012510. 